\title{
Facilitating the integration of emotional states in patients' personal disease experience with cancer: a new brief intervention for managing psychological distress
}

\author{
Maria Antonietta Annunziata • Barbara Muzzatti • \\ Lorena Giovannini - Sara Mella • Francesca Bomben • \\ Cristiana Flaiban • Andrea Veronesi
}

Received: 27 August 2012 / Accepted: 28 January 2013 / Published online: 9 February 2013

(C) Springer-Verlag Berlin Heidelberg 2013

\begin{abstract}
Purpose This paper describes an intervention performed at an Italian oncological institute to manage psychological distress related to the oncological experience. Its objectives are to encourage and normalize awareness of the importance of emotional aspects of the cancer experience, to provide psycho-education to patients on the importance of psychosocial care in promoting well-being, and to introduce our psychology service and promote its usage.

Methods The intervention consists of three consecutive steps: the psychological distress screening; the clinical interview, which is conducted according to Rogers' clientcentered model; and the collection of data regarding the appreciation and usefulness of the initiative, performed through a feedback questionnaire and the codification of the interview contents.

Results Between September 2011 and February 2012, the intervention was administered to 484 consecutive new inpatients. Among them, the prevalence of psychological distress and its components of anxiety and depression are comparable to those found in the literature. The low percentage of participants who refuse the screening (15.4\%) as well as of those who do not wish to have the results returned to them (3.1\%),
\end{abstract}

M. A. Annunziata $\cdot$ B. Muzzatti $\cdot$ L. Giovannini $\cdot$ S. Mella $\cdot$

F. Bomben $\cdot$ C. Flaiban

Unit of Oncological Psychology, Centro di Riferimento

Oncologico IRCCS Istituto Nazionale Tumori, Aviano,

Pordenone, Italy

A. Veronesi

Division of Medical Oncology C, Centro di Riferimento

Oncologico National Cancer Institute, Aviano, Italy

M. A. Annunziata ( $\square)$

Centro di Riferimento Oncologico, IRCCS Istituto

Nazionale Tumori, Via F. Gallini, 2,

33081 Aviano, Pordenone, Italy

e-mail: annunziata@cro.it together with the high scores regarding the usefulness and effectiveness given to the intervention (all $>80 / 100$ ), documents the positive reception of this activity. Lastly, the analysis of the contents of the exit interview shows that a wide range of themes, far more varied and heterogeneous than just anxiety and/or depression symptoms, was discussed.

Conclusions Even though this was a clinical and not a research activity, it still offers important descriptive data.

Keywords Assessment · Cancer · Psychological distress · Oncology $\cdot$ Psychological intervention

\section{Introduction}

Cancer presents itself as a pervasive and multi-faceted experience of suffering. It is, indeed, difficult to limit it simply to the affected area of the body because it involves the whole organism, as well as the psychological and social sphere. The term "psychological distress" is purposely "wide-ranging" and removed from the stigmatizing terminology that is used in psychiatry and is meant to encompass all the different forms of discomfort related to the oncological experience, whether they be mild or severe, transitory or permanent [1].

In North America and western Europe, the prevalence of psychological distress is between 22 and $45 \%$ [2]. When we specifically look at the two most commonly studied components of psychological distress (depression and anxiety), we can see that the prevalence of depression is between 9 and $21 \%$, while the prevalence of anxious states is between 10 and $48 \%$. Furthermore, several studies have demonstrated that clinically significant distress is associated with maladaptive coping, more needs, reduction in quality of life, impairment of social relationships, risk of suicide, longer rehabilitation time, poor adherence to treatment, abnormal 
illness behavior, family dysfunction, psycho-social morbidity, and possibly shorter survival [3-7].

All of these justify the fact that psycho-social care is considered a fundamental aspect of quality oncological care [8] and, in particular, that constant monitoring and management of psychological distress is widely recommended [9]. Unfortunately, less than $10 \%$ of the cases are actually recognized and referred to mental health services [10]. This lack of recognition of distress causes problems for the patients (regarding making therapeutic decisions, complying to treatments, and making complementary medical appointments), but it also cause problems for the oncological team, in terms of stress and length of appointment times [9, 11, 12].

In this paper, we describe how oncology-related psychological distress was screened and managed at an Italian oncological institute. The objectives were as follows: (1) to encourage/normalize awareness of the importance of emotional aspects of the cancer experience, (2) to provide psychoeducation to patients on the importance of psycho-social care in promoting well-being, and (3) to introduce the patient to the institute's psychology service and to promote its usage.

\section{Materials and methods}

Sample and setting

From September 2011 to February 2012, at the Centro di Riferimento Oncologico di Aviano (the National Cancer Institute in north-eastern Italy), a clinical intervention dedicated to helping patients in integrating emotional states in their own disease was systematically started. Thus, all new inpatients (i.e., those who were in the diagnostic phase or were receiving their first treatment) of both medical and surgical divisions were offered to receive this intervention by a trained psychologist.

\section{The intervention}

The main objective of the intervention consisted in helping patients to acknowledge and integrate emotions as natural aspects of their disease experience so that their multidimensional (bio-psycho-social) well-being can become an achievable goal for all of them. In other words, the intervention was directed at encouraging cancer patients to also pay attention to their emotional states and start considering them as an object for care just as much as their bodies.

The intervention consisted of three consecutive steps: (1) the psychological distress screening, (2) the clinical interview, and (3) the collection of data regarding the appreciation and usefulness of the intervention.

In the first step, a psychologist contacted the patients and presented the intervention as a routine practice of the institute which measures and monitors emotions, just like routine checks of blood pressure, temperature, pulse, breathing, and pain. The psychologist delivered and accurately described the screening instruments in order to ensure proper autonomous completion and informed patients that the results would be discussed in a subsequent meeting. The administered instruments were the hospital anxiety and depression scale (HADS [13]), and the distress thermometer and problem list (DT-PL [14]). The HADS is a 14-item, self-report measure of anxiety (seven items) and depression (seven items). For each item, respondents are asked to indicate which of the four options (rated 3-0) comes closest to describing how they have been feeling. Scores between 8 and 10 indicate possible cases, scores between 11 and 21 probable cases in both scales. The DT is a visual analogue tool asking participants to rate their level of distress in the week prior to the interview on a scale from 0 to 10 (from " 0 no distress" to " 10 extreme distress"). Scores between 0 and 4, 5 and 7, and 8 and 10 indicate, respectively, absent, moderate, and severe psychological distress. The PL consists of a list of 36 problems patients may experience grouped into five categories (practical problems, family problems, emotional problems, spiritual/religious concerns, and physical problems) and that are rated in a yes/no format. Patients are contacted when they first enter the hospital division. The intervention was proposed the day after admission. Because of the complexities related to the operative phases (e.g., pre-operative anxiety, post-surgery physical conditions), in the breast surgery division, the activity was proposed the day after surgery, whereas in the general or gynecological oncological surgery division, it was proposed 3 days after the operation. The first step did not require any specific written agreement from participants because it is included in the institute's standard clinical practice. The patient, however, may refuse both the psychological distress screening and the following meeting.

The second step took place the same day, if the distress screening had been performed in the morning, or the next day, if the screening had been conducted in the afternoon, depending on the level of patient distress, the follow-up meeting could be limited to simply returning results or expanded to a more in-depth clinical interview. When distress seemed to be absent, a brief follow-up meeting was provided to patients. During this meeting, they were encouraged to express any potential needs and were encouraged to contact the oncological psychology unit at any time in the future if they felt the need to speak to someone. When the distress was moderate or severe, the objectives became more complex. In particular, patients were encouraged to understand the disease experience through speaking about its subjective meaning and to acknowledge and understand the emotions they expressed. They were also made aware of suffering and its causes, given information which could be useful in the practical management of cancer (e.g., where 
information materials are available about who gives insurance and pension information or information about volunteer organizations), and, ultimately, motivate to participate in a more structured professional intervention. In this second case, a complete clinical interview was performed; it was conducted in a non-directive way and based on client-centered therapy as developed by Rogers [15]. Acceptance of the other as an individual, absence of judgment about the issues being discussed, empathy, and the ability "to see through the patient's eyes" are the main elements of the interview that allow an understanding of the patient's actual disease experience by the psychologist. Both verbal and non-verbal communications were used together with paraphrasing. The clinical interview lasted $20 \mathrm{~min}$ on average.

In the third step, which was directed at gathering data regarding the usefulness and appreciation of the initiative, two different activities were performed: on one hand, the psychologists who were conducting the intervention codified the themes discussed in the interviews according to a series of keywords that had previously been identified; on the other hand, a small sub-sample of patients (consisting of patients admitted at the institute in a pre-established week who had received the clinical interview) was asked to evaluate the intervention they had received by filling out an anonymous feedback questionnaire. This consists of four ad hoc questions designed to investigate on a scale of 1-100: the degree to which participants felt listened to and understood by the psychologists, the usefulness of the interview with respect to clarifying their situation, the participants' level of emotional well-being after the interview, and the usefulness of the interview; in addition, suggestions, comments, and requests can be added in a specific section. This questionnaire was given to patients at the end of the interview, and they were asked to fill it out whenever they could before leaving it in a dedicated box situated in the unit's hallway.

The intervention was carried out by three psychologists (S.M., F.B., C.F.) who are trained in the management of cancer's psycho-emotional aspects and have been working in oncology for at least 2 years. The three psychologists who administered the intervention were previously trained by three psychotherapists (M.A.A., B.M., L.G.) in paraphrasing, empathy, and in keeping non-judgmental attitudes in a 2-day training. Furthermore, during the first 2 weeks, the psychologists' intervention administrations were discussed and supervised in the group, to further standardize the approach to the patient as well the intervention administration.

\section{Results}

In the period mentioned, the intervention was offered to 572 inpatients admitted to our institute for the first time. Because 88 subjects $(15.4 \%)$ declined to participate, the final sample was of 484 persons. Among them, $20.9 \%$ were males, $72.3 \%$ were partnered, and the median age was 54 years (range, 22-87). Of the sample, $49.7 \%$ reported having 8 years of education (i.e., the compulsory education in Italy), 38.6\% reported having a post-compulsory education, and $11.7 \%$ reported having a post-secondary degree. Looking at their professional status, $55.1 \%$ were employed, whereas $44.9 \%$ were unemployed, retired, students, or housewives. Lastly, $37.9 \%$ of the sample were inpatients in a medical ward, whereas $62.1 \%$ were in a surgical ward.

We found probable levels of anxiety and depression in, respectively, $24.2 \%$ and $15 \%$ of the sample. When we add patients with possible anxiety or depression, the percentages rise to 50.1 and $30 \%$, respectively. Regarding psychological distress, as measured by the distress thermometer, it is normal in $41 \%$ of the sample, moderate in $26.4 \%$, and severe in $32.6 \%$. On a scale of $0-100$, the average of the problems for each category is $24.2(\mathrm{SD}=22.7)$ for emotional problems, 12.8 $(\mathrm{SD}=11.9)$ for physical problems, $10.1(\mathrm{SD}=19.1)$ for relational problems, $10.0(\mathrm{SD}=14.2)$ for practical problems, and $5.4(\mathrm{SD}=22.7)$ for spiritual or religious problems. At the end of the intervention, $5.9 \%$ of participants accepted the suggestion or expressed interest to participate in a more structured psychological support.

Forty six participants $(9.5 \%$ of the sample) were asked to complete the feedback questionnaire. On a 1-100 anchored evaluation scale, the subjects quantified the following items: the degree to which they felt listened to by the provider, with an average score of $93.3(\mathrm{SD}=8.2)$; the usefulness of the interview in regards to clarifying their situation, with an average score of $81.7(\mathrm{SD}=14.0)$; their level of emotional well-being after the interview, with an average score of 81.8 $(\mathrm{SD}=12.5)$; and the usefulness of the interview with an average score of $91.1(\mathrm{SD}=9.2)$. Only 2 out of 46 subjects $(4.3 \%)$ used the available space to write their own comments, and those were both declarations of gratitude for this activity.

In $78.4 \%$ of the cases, the psychologist offered the participants a brief follow-up meeting to return the results, while in the rest of cases, $21.6 \%(N=98)$, the psychologist offered a longer follow-up encounter (i.e. the clinical interview). Table 1 illustrates the frequency and total percentage of the themes discussed during the clinical interview, following a previously established codification.

\section{Discussion}

This paper describes a routine clinical activity performed, in accordance to the international guidelines [1, 8, 9], in order to inform oncological patients of the importance to take care of their emotions (as well as their diseased bodies) and also to alert them to the presence of the psychology service as a tool that facilitates holistic self-care. Considering that this is 
Table 1 Themes discussed in the clinical interview: frequencies and percentages on total $(N=98)$

\begin{tabular}{lccc}
\hline Themes & Number & \% on total items & \% on total patients \\
\hline Relationships (family, couple, friends) & 51 & 18.7 & 52.0 \\
Anxiety symptoms & 49 & 17.9 & 50.0 \\
Disease (waiting for test results, therapies, & 48 & 17.6 & 49.0 \\
$\quad$ prognosis, side effects, relapses, hospitalization, & & & \\
$\quad$ being far from home) & 30 & 11.0 & 40.6 \\
Emotions and their management & 27 & 9.9 & 27.6 \\
Ordinary activities management (work, family, & & & 15.3 \\
$\quad$ routine, need of normality, changes) & 15 & 5.6 & 13.3 \\
Existential or spiritual aspects & 13 & 4.8 & 12.2 \\
Previous or current stressful events (family members & & & 11.2 \\
$\quad$ diseases, deaths, separation, pre-existing diseases) & 12 & 4.4 & 8.2 \\
Depressive symptoms & 11 & 4.0 & 5.1 \\
Future and death & 8 & 2.9 & 2.0 \\
Communication with personnel & 5 & 1.8 & 2.0 \\
Body image & 2 & 0.7 & - \\
Money worries & 2 & 0.7 & \\
Voluntary cancer work & 273 & 100.0 & \\
Total & & & \\
\hline
\end{tabular}

a clinical activity, it does not have the advantages and merits of well-constructed experimental design; in spite of this, though, it offers important descriptive data for various types of providers who take care of oncological patients.

First of all, the prevalence of psychological distress, as well as forms of anxiety and depression we have found, is similar to the levels found in the literature for other samples. Considering the patients that were involved in this study are in the diagnostic phase or just starting treatment, these data support the idea that the phenomenon of psychological distress is already evident at this time.

Secondly, the activity is appreciated and has high participation numbers. The low percentage of subjects who refuse screening, just like the negligible percentage of those who do not wish to have the results of their screenings returned to them, together with the high scores given to the intervention as far as usefulness and effectiveness in the feedback questionnaire, all point to the positive reception of the activity.

The proportion of people who were offered the brief follow-up meeting (limited to returning the results of the interview materials) and those who received the clinical interview, is about equal to the proportion of subjects with no to mild distress and those who exhibited more symptoms.

Particularly, the analysis of the contents of the clinical interview is very informative because it shows that a wide variety of themes were discussed. Apart from anxiety and depression symptoms, many other issues came up during the clinical interview. Some patients spoke about issues with different types of relationships. Others expressed the need to speak more about the illness and its medical and practical aspects (even with a provider who was not directly involved in therapy management and medical communications).
Participants discussed the actual impact of the illness on their functioning on the job, in the family, etc. They spoke about other current or past stressful life events, of body image, of the future and death, of spirituality, and also of the difficulties they encountered in communicating with healthcare providers. The welcoming listening techniques used by the psychologists during the clinical interview seem to facilitate the emergence of the complex and heterogeneous elements of worry and difficulty that influence the emotional state of the individual, as well as the subjective experience of the illness. In this sense, this descriptive information seems much more in sync with the complexity of the subjective experience of the illness, rather than the needs profile that surfaced from the problem list paired with the DT. Furthermore, the fact that the clinical interview encompassed so many different issues, in addition to the fact that the initiative was very well received, seems to point to the usefulness of this experience for the patients' well-being.

The limitation of this study is that this is a description of a clinical activity. As already discussed, this was not an experimental verification of an intervention model, so we cannot come to conclusions regarding its effectiveness. In particular, the small number of patients requested to fill out the feedback questionnaire and the small percentage of coded interviews provide only descriptive information. Nonetheless, the acceptance and participation (also in terms of the amount of issues that came to light) definitely document the positive response of patients regarding a brief activity that was meant to recognize and welcome their emotional state regarding their oncology experience. In addition, it should be recognized that this intervention, though inexpensive in terms of resources, reaches all patients during their first admission to the hospital and 
allows all of them to be well-informed regarding the multidimensional aspects of the illness. Furthermore, another strength of this intervention is the fact that it can be translated to different healthcare settings.

In order to attempt to capture the effectiveness of a standardized and replicable psychological intervention, certain methods have to be applied. For this to happen, it would have been necessary to have indices of pre- and post-intervention distress levels to compare data to a control group which is not offered any activity and to appropriately check the heterogeneity of the sample (both as far as socio-demographic as well as for as clinical factors go). Our data simply indicate a positive response and evaluation on the part of oncological patients for such an activity. Future research will have to determine its effectiveness in objective terms.

Acknowledgments The authors wish to thank Ms Anna Vallerugo, MA, for her writing assistance.

Conflict of interest All authors declare that they have no conflict of interest relevant to this manuscript. Authors have full control of all primary data and agree the journal to review these data if requested.

\section{References}

1. Network NCC (1999) Distress management. Clin Pract Guidelines $1: 344-374$

2. Annunziata MA, Muzzatti B (2012) Psychological distress in oncology. In: Ohayashi H, Yamada S (eds) Psychological distress: symptoms, causes and coping. Nova Publisher, Hauppauge, pp 27-48
3. Ballenger JC, Davidson JRT, Lecrubier Y, Nutt DJ, Jones RD, Berard RMF, International Consensus Group on Depression and Anxiety (2001) Consensus statement on depression, anxiety and oncology. J Clin Psychiatry 62(suppl 8):64-67

4. Grassi L, Riba M (2009) New frontiers and challenges of psychiatry in oncology and palliative care. In: Christodoulou GN, Jorge M, Mezzich JE (eds) Advances in psychiatry (vol 3). Beta Medical, Athens, pp 105-114

5. Hamer M, Chida Y, Molloy GJ (2009) Psychological distress and cancer mortality. J Psychosom Res 66:255-258

6. Bringman H, Singer S, Höckel M, Stolzenburg JU, Krauss O, Schwarz R (2008) Longitudinal analysis of psychiatric morbidity in cancer patients. Onkologie 31:343-344

7. Annunziata MA, Muzzatti B, Bidoli E, Veronesi A (2012) Emotional distress and needs in Italian cancer patients: prevalence and associations with socio-demographic and clinical factors. Tumori 98:132-138

8. Adler NE (2008). Cancer care for the whole patient: meeting psychosocial health needs. Institute of Medicine (IOM). eds. The National Academies Press, Washington, DC

9. National Comprehensive Cancer Network (2011) Distress management. Clinical practice guidelines in oncology, version 2011

10. Kadan-Lottick NS, Vanderwerker LC, Block SD, Zhang B, Prigerson HG (2005) Psychiatric disorders and mental health service use in patients with advanced cancer: a report from the Coping with Cancer study. Cancer 104:2872-2881

11. Zabora JR (1998) Screening procedures for psychosocial distress. In: Holland JC, Breibart W, Jacobsen PB et al (eds) Psychooncology. Oxford University Press, New York, pp 653-661

12. Carlson LE, Bultz BD (2003) Cancer distress screening. Needs, models, and methods. J Psychosom Res 55:403-409

13. Zigmond AS, Snaith RP (1983) The hospital anxiety and depression scale. Acta Psychiatr Scand 67:361-370

14. Roth AJ, Kornblith AB, Batel-Copel L, Peabody E, Scher HI, Holland JC (1998) Rapid screening for psychologic distress in men with prostate carcinoma. Cancer 82:1904-1908

15. Rogers C (1951) Client-centered therapy. Houghton Mifflin, Boston 